\title{
Degos' disease: association with anticardiolipin antibodies and the lupus anticoagulant
}

\author{
H J ENGLERT, C H HAWKES, M L BOEY, G J M DERUE, S LOIZOU, E N HARRIS, \\ A E GHARAVI, R G HULL, G R V HUGHES
}

\begin{abstract}
A woman presented with multiple cerebral thromboses and skin lesions characteristic of Degos' disease. Her serum contained high titres of anticardiolipin antibodies and showed lupus anticoagulant activity, both known to be strong markers of a thrombotic tendency in systemic lupus erythematosus. This finding may have therapeutic implications for this usually fatal disease.
\end{abstract}

\section{Introduction}

Degos' disease, a rare multisystemic vasculopathy, is characterised by widespread thrombosis, particularly of the vessels of the skin, central nervous system, and gastrointestinal tract. ${ }^{1}$ Once systemic disease occurs death usually follows due to the effects of multiple cerebral or bowel infarcts. ${ }^{1}$ The aetiology is unknown. At necropsy the vessels show little evidence of inflammation, the predominant lesion being widespread thrombosis, particularly within medium and small arteries and arterioles. We report on a patient with the disease.

\section{Case report}

A 36 year old woman first noted painless skin lesions in early 1978 . A year later Degos' disease was diagnosed. In 1980 she developed loss of pain and temperature sensation in a TR-L1 distribution. Ischaemic myelopathy was considered to be most likely, and treatment with aspirin and dipyridamole was started. In 1982 she suddenly developed occlusion of the central retinal artery with bilateral choroidal infarcts, and over the ensuing 12 months she developed a series of new neurological features, with episodes of ataxia, difficulty in speaking, bowel and bladder incontinence, and weakness and numbness of both legs. On referral to the rheumatology unit at Hammersmith Hospital she was again noted to have the skin lesions of Degos' disease. Neurological abnormalities included a distended bladder and lax anal tone, bilateral pyramidal abnormalities, and sensory loss to pain and light touch to the level of $\mathrm{T} 8$. The following investigations were normal : full blood count, antinuclear antibody titre, immunoglobulin concentrations, rheumatoid factor titre, complement profile, tests for immune complexes, vitamin $\mathbf{B}_{12}$ assay, prothrombin time, partial thromboplastin time, and skull $x$ ray films. The erythrocyte sedimentation rate was $40 \mathrm{~mm}$ in the first hour. A Venereal Disease Research Laboratory test was positive at $1 / 4$ and a Treponema pallidum haemagglutination test was negative. A repeat myelogram was normal. Lumbar puncture showed a raised protein concentration of $1.9 \mathrm{~g} / \mathrm{l}$. Skin biopsy showed areas of collagen necrosis and atrophy of the epidermis and a conspicuous

\footnotetext{
Rheumatology Unit, Department of Medicine, Royal Postgraduate

Medical School, Hammersmith Hospital, London W12 0HS

H J ENGLERT, MB, Bs, honorary clinical assistant

M L BOEY, MMED, honorary clinical assistant

G J M DERUE, MD, honorary clinical assistant

$S$ LOIZOU, MSC, senior scientific officer

E N HARRIS, DM, DPHIL, research fellow

A E GHARAVI, MD, honorary lecturer

R G HULL, MB, MRCP, honorary registrar and research fellow

G R V HUGHES, MD, FRCP, consultant physician and reader in rheumatology

Ipswich Hospital, Ipswich

C H HAWKES, MD, MRCP, consultant neurologist

Correspondence to: Dr H J Englert.
}

absence of inflammatory cells at the margin of the lesion. The presence of the lupus anticoagulant was shown by a previously described method. ${ }^{2}$ Anticardiolipin antibodies were measured by the method of Harris et al. ${ }^{3}$

Treatment with daily prednisolone and azathioprine together with intermittent pulse cyclophosphamide was started. While the anticardiolipin antibody titre was still raised, however, she developed a further spinal cord lesion at $\mathrm{C} 8$, which led to her eventual death.

\section{Discussion}

We recently reported the strong association between the presence of anticardiolipin antibodies and the lupus anticoagulant and a tendency to thrombosis. ${ }^{3}$ Of particular interest was the presence of raised anticardiolipin antibody titres in 13 of 15 patients with systemic lupus erythematosus and related autoimmune disorders who had cerebral infarction. Although our studies so far have concentrated on systemic lupus erythematosus, the association may possibly also extend to other diseases. In preliminary studies nine of 25 patients with Beçhet's syndrome (a disease characterised by both thrombosis and cerebral disease and a notable absence of circulating antibodies) raised titres of anticardiolipin antibodies were detected (unpublished observations).

The mechanisms by which anticardiolipin antibodies lead to thromoosis are unclear; inhibition of production of prostacyclin by endothelial cells may possibly be important. ${ }^{4}$ Whether suppression of the production of antiphospholipid antibodies will result in clinical benefit is not yet known, although of interest is the preliminary communication of Lubbe et al who treated six patients with systemic lupus erythematosus who had had repeated spontaneous abortions and in whom the lupus anticoagulant was present. ${ }^{5}$ The lupus anticoagulant was suppressed oy treatment with prednisolone and aspirin, and in five of the six patients successful pregnancies were possible.

The pathogenesis of Degos' disease is unknown. The main pathological feature is damage to endothelial cells in the small arteries and arterioles, with associated thrombosis and partial or complete obliteration of the vascular lumen. We believe that the presence of the lupus anticoagulant and high titres of anticardiolipin antibodies in a patient with Degos' disease may have aetiological implications. It might also provide a basis for treatment of this fatal disease.

We thank Dr Lyons, Dr Alexander, Dr Chisholm, Mr Seys, and $\mathrm{Mr}$ Burgmeister for their help in the management of this case, and Miss S Lydon for secretarial help.

\section{References}

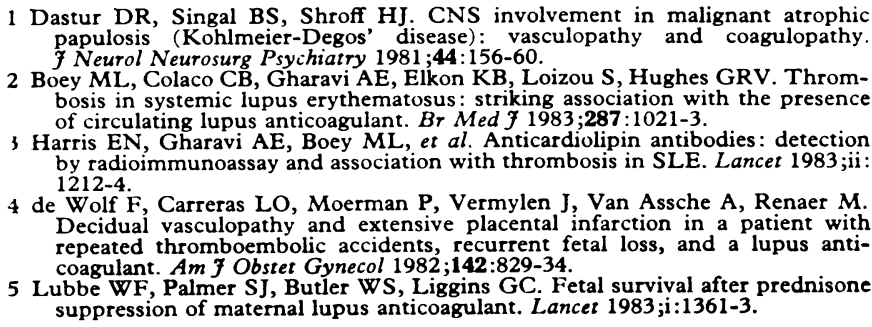

